\title{
Randomized, placebo-controlled, adjunctive study of armodafinil for bipolar I depression: implications of novel drug design and heterogeneity of concurrent bipolar maintenance treatments
}

Mark A Frye ${ }^{1 *}$, Jess Amchin ${ }^{2}$, Michael Bauer ${ }^{3}$, Caleb Adler ${ }^{4}$, Ronghua Yang ${ }^{2}$ and Terence A Ketter ${ }^{5}$

\begin{abstract}
Background: Some, but not all, prior investigations suggest armodafinil may have utility as an adjunctive treatment in bipolar I depression.

Methods: Multicenter, randomized, double-blind study in patients aged 18 to 65 years experiencing a depressive episode despite maintenance therapy for bipolar I disorder. Patients were randomized to receive adjunctive armodafinil $150 \mathrm{mg} /$ day or adjunctive placebo for 8 weeks. Primary efficacy outcome was change from baseline in 30-Item Inventory of Depressive Symptomatology-Clinician-Rated (IDS- $C_{30}$ ) total score at week 8 . Safety and tolerability were monitored.

Results: Of 656 patients screened, 399 were randomized, of whom 308 (77\%) were taking a protocol-allowed mood stabilizer as monotherapy. The primary efficacy outcome did not reach statistical significance; however, several secondary efficacy outcomes demonstrated statistically significant advantages for adjunctive armodafinil $(n=197)$ over adjunctive placebo $(n=196)$, including Clinical Global Impression of Severity of Illness for depression (weeks 6, 8, and endpoint; all $P<0.05$ ), Global Assessment of Functioning (weeks 4, 8, and endpoint; all $P<0.02$ ), IDS- $C_{30}$ remitter rates (week 8 and endpoint; both $P<0.02$ ), and mean change from baseline in IDS- $C_{30}$ total score at week $7(P<0.05)$. Adjunctive armodafinil and adjunctive placebo were generally well tolerated. Although adjunctive armodafinil compared with adjunctive placebo yielded a higher headache rate (15 vs $8 \%$ ), it yielded similar (generally favorably low) rates of all-cause discontinuation (16 vs $16 \%$ ), adverse event discontinuation ( 4 vs $5 \%$ ), nausea ( 6 vs $4 \%$ ), $\geq 7 \%$ weight gain ( 2 vs $5 \%$ ), anxiety (4 vs $3 \%$ ), insomnia (3 vs $2 \%$ ), sedation/somnolence (1 vs $1 \%$ ), and hypomania (0 vs $<1 \%$ ).
\end{abstract}

Conclusions: In this study, adjunctive armodafinil compared with adjunctive placebo in bipolar I depression did not separate in the primary efficacy outcome but demonstrated advantages for several secondary efficacy outcomes and was generally well tolerated. Additional research is warranted and necessary to better identify clinical predictors (e.g., atypical depressive symptoms, specific anti-manic/mood-stabilizing agents used) that would provide optimized, individualized therapeutics for bipolar depression.

Trial registration: ClinicalTrials.gov: NCT01305408

Keywords: Armodafinil; Bipolar I disorder; Major depressive episode

\footnotetext{
* Correspondence: mfrye@mayo.edu

'Department of Psychiatry, Mayo Clinic, 200 First Street SW, Rochester, MN 55901, USA

Full list of author information is available at the end of the article
} 


\section{Background}

Bipolar disorder is a recurrent and debilitating illness that affects approximately $2.6 \%$ of the adult population (Kessler et al., 2005), with approximately $1 \%$ of the population having bipolar I disorder (Merikangas et al., 2007). Depression is the predominant and prevailing symptomatic illness phase. For example, in bipolar I disorder, depression accounts for at least three times more time ill than mania (Judd et al., 2002; Kupka et al., 2007). Depressive episodes are associated with more disability, comorbidity, and functional and occupational deficits than manic episodes; moreover, the depressive phase is associated with an increased risk of recurrence of mood symptoms and rates of suicide (Bauer et al., 2001; Calabrese et al., 2004; Merikangas et al., 2007; Merikangas et al., 2011). In clinical settings, adjunctive treatments may be added to maintenance therapies when mood symptoms recur, but the clinical evidence base is limited in this type of therapeutic intervention. Only one agent, lurasidone, has been approved by the United States Food and Drug Administration (FDA) as adjunctive treatment, combined with either lithium or valproate for acute bipolar I depression (Latuda [package insert], 2013). FDA-approved treatments for acute bipolar I depression include the olanzapine/fluoxetine combination, quetiapine monotherapy, and lurasidone. Investigations of aripiprazole monotherapy (Thase et al., 2008) and ziprasidone, as both monotherapy and adjunctive therapy (Lombardo et al., 2012; Sachs et al., 2011), failed to provide significant improvements in depressive symptoms compared with placebo in bipolar I depression. Clearly, given the morbidity of bipolar depression, more treatment options are needed.

Armodafinil ( $R$-modafinil) is a wakefulness-promoting low-affinity dopamine transport inhibitor that is currently approved in the USA for the treatment of excessive sleepiness associated with shift work disorder, narcolepsy, and obstructive sleep apnea (Nuvigil [package insert], 2013). Preliminary research on modafinil and armodafinil suggested potential benefit in mood disorders (Calabrese et al., 2010; Calabrese et al., 2014; Frye et al., 2007).

An initial phase 3 randomized, placebo-controlled study of adjunctive armodafinil $150 \mathrm{mg} /$ day demonstrated a significant benefit in bipolar I depression on the primary outcome (mean change from baseline in the 30-Item Inventory of Depressive SymptomatologyClinician-Rated (IDS- $\mathrm{C}_{30}$ ) total score) (Rush et al., $2000)$ in comparison with placebo $(P<0.01)$, as well as some secondary outcomes (Calabrese et al., 2014). In contrast, a second, similarly designed phase 3 study found that adjunctive armodafinil $150 \mathrm{mg} /$ day provided numerically, but not statistically significantly, greater improvement in bipolar I depression than placebo on the same primary outcome (Ketter et al., 2015).
The current study (ClinicalTrials.gov, study identifier NCT01305408) was performed to further investigate the efficacy, safety, and tolerability of adjunctive armodafinil use in bipolar I depression. As in prior investigations, this study permitted adjunctive armodafinil or adjunctive placebo to be combined with a broad array of ongoing bipolar disorder maintenance treatments, in conformity with the availability of many such choices in clinical practice, providing the strength of increased generalizability, albeit with the potential limitation of decreased assay sensitivity. This study, unlike prior studies, included quetiapine as one of the permitted maintenance therapies, further contributing to this study's generalizability and relevance to clinical practice. As in prior studies, efficacy was assessed by the mean change from baseline in IDS- $\mathrm{C}_{30}$ total score.

\section{Methods}

This phase 3, 8-week, randomized, double-blind, placebo-controlled, parallel-group, fixed-dosage, multicenter study was conducted at 84 centers in 13 countries across 4 regions. Patients who were experiencing a major depressive episode, despite stable doses of protocol-allowed "mood stabilizers" (lithium and certain anticonvulsants and antipsychotics) for the treatment of bipolar I disorder, were randomly assigned to adjunctive armodafinil $150 \mathrm{mg} /$ day or matching placebo in a 1:1 ratio. The 150-mg dose of armodafinil was selected based on efficacy and tolerability data from previous clinical trials. Randomization was stratified on the basis of concomitant mood stabilizers being taken (lithium, anticonvulsants, and antipsychotics). If a patient was taking more than one of these medications at randomization, the patient was assigned to the category of the medication of longest duration at the discretion of the investigator. Randomization was also stratified by region of the world (Region 1, the USA and Canada; Region 2, Armenia, Azerbaijan, Belarus, Bulgaria, Georgia, Russia, Ukraine, Kyrgyzstan, Mongolia, Uzbekistan, Albania, Bosnia, Herzegovina, Croatia, Cyprus, Czech Republic, Greece, Hungary, Poland, Serbia, Slovakia, Slovenia, Republic of Macedonia, and Turkey; Region 3, Andorra, Austria, Australia, Belgium, Denmark, Finland, France, Germany, Greece, Iceland, Ireland, Italy, Liechtenstein, Luxembourg, Malta, Monaco, the Netherlands, Norway, Portugal, San Marino, Spain, Sweden, Switzerland, the UK, and Vatican City; Region 4, rest of the world).

\section{Ethics, consent, and permissions}

The study was conducted in accordance with the International Conference on Harmonisation's Guideline for Good Clinical Practice, and the study protocol and amendments were approved by the independent ethics committee/institutional review board at each participating 
center. Written informed consent was obtained from each patient before screening.

\section{Participants}

Inclusion and exclusion criteria were similar to those reported in detail for the previous two studies (Calabrese et al., 2014; Ketter et al., 2015). Specifically, patients were aged 18 to 65 years and had bipolar I disorder with current non-psychotic depression according to the Diagnostic and Statistical Manual of Mental Disorders (Fourth Edition, Text Revision; DSM-IV-TR) criteria (American Psychiatric Association, 2000), as determined by the Structured Clinical Interview for DSM-IV, Clinical Trials (SCID-CT) (First et al., 2007). Patients were required to have had $\geq 1$ previous manic or mixed episode, which resulted in functional impairment that was treated (or should have been treated) with a protocolallowed mood stabilizer. Patients could not have had $>6$ mood episodes in the prior year and their current depressive episode must have started $\geq 2$ weeks but $\leq 12$ months prior to the screening visit and occurred despite taking stable doses of one or two mood stabilizers, defined specifically as lithium, valproate, lamotrigine, olanzapine, quetiapine, aripiprazole, risperidone, or ziprasidone (ziprasidone only in combination with lithium, valproate, or lamotrigine). In patients taking two mood stabilizers, one was required to be lithium, valproate, or lamotrigine. Medications known to induce CYP3A4/5, such as carbamazepine, were not permitted within 14 days before the baseline visit or during the study. Furthermore, onset of the current depressive episode had to be $\geq 8$ weeks after resolution of any previous mood episode. Concomitant antidepressant use was not allowed within 14 days or five half-lives before study entry or during the study. This exclusion was exercised to minimize the confounding of any effects seen regarding adjunctive drug-placebo differences in bipolar I depressive symptom improvement with armodafinil.

Patients were required to have had screening and baseline 16-Item Quick Inventory of Depressive Symptomatology-Clinician-Rated (QIDS-C 16 ) (Rush et al., 2003) scores $\geq 13$, Clinical Global Impression of Severity of Illness (CGI-S) for depression score $\geq 4$, Young Mania Rating Scale (YMRS) (Young et al., 1978) total score $\leq 10$, and YMRS scores of 0 or 1 on items 1 to 3 . Patients were also required to be in good health based on a physical examination, electrocardiogram, and laboratory studies.

Patients were excluded if they had other Axis I disorders within 6 months of screening that were the focus of treatment, or Axis II disorders of concern (borderline, antisocial, or other personality disorders that could impact conduct of the study), a history of alcohol or substance abuse or dependence (with the exception of nicotine dependence) within 3 months of the screening visit or during the screening period, current psychotic symptoms or psychosis within 4 weeks of screening, active suicidal ideation or history of significant suicidal behaviors, score of $\geq 2$ on item 18 of the IDS- $\mathrm{C}_{30}$, Hamilton Anxiety Scale (HAM-A) (Hamilton Anxiety Rating Scale, 2011) total score $\geq 17$ at baseline, or a history of clinically significant cutaneous drug or hypersensitivity reactions.

\section{Assessments}

The primary efficacy assessment was the mean change from baseline assessed at week 8 in total IDS- $\mathrm{C}_{30}$ score for adjunctive armodafinil $150 \mathrm{mg} /$ day versus adjunctive placebo as analyzed by mixed-model repeated measures (MMRM). Secondary efficacy assessments included mean change from baseline in the IDS- $C_{30}$, QIDS- $C_{16}$, and CGI-S, as well as IDS- $\mathrm{C}_{30}$ response $(\geq 50 \%$ reduction from baseline in total score), IDS- $C_{30}$ remission (final IDS- $\mathrm{C}_{30}$ score $\leq 11$ ) rates, and CGI-S response (decrease $\geq 2$ points in severity from baseline) rate, all assessed at weeks $1,2,4,6,7$, and 8 (or early termination), as well as mean change from baseline in the Global Assessment of Functioning (GAF), assessed at weeks 4 and 8 , or early termination.

Safety assessments included mean change from baseline in the YMRS and the Columbia-Suicide Severity Rating Scale-Since Last Visit (C-SSRS-SLV) (Posner et al., 2009) at weeks $1,2,4,6,7$, and 8 (or early termination), mean change from baseline in the HAM-A and Insomnia Severity Index (ISI) (Bastien et al., 2001) at weeks 4 and 8 (or early termination), as well as collection of adverse events (AEs) and serious AEs (SAEs), vital signs, and laboratory studies.

\section{Statistical analysis}

Sample-size calculations were based on IDS- $\mathrm{C}_{30}$ total score and used estimates of variability obtained from previous phase 2 and 3 results for armodafinil and modafinil investigations in bipolar I depression (Calabrese et al., 2010; Calabrese et al., 2014; Frye et al., 2007). Target enrollment was 370 patients (185 patients for each of adjunctive armodafinil $150 \mathrm{mg} /$ day and adjunctive placebo) to ensure that at least 332 patients (166 per group) were evaluable for efficacy, providing $85 \%$ power to detect a mean between-group difference of 4 points in the change from baseline in IDS- $\mathrm{C}_{30}$ total score (assuming a standard deviation of 12.1). Data from 393 and 398 patients were analyzed for efficacy and safety, respectively.

Patients receiving $\geq 1$ dose of study drug were analyzed for safety (safety analysis set), and patients in the safety analysis set who had $\geq 1$ post-baseline IDS- $\mathrm{C}_{30}$ efficacy assessment were analyzed for efficacy (full analysis set). For the primary outcome, IDS- $\mathrm{C}_{30}$ total score was analyzed 
using MMRM as previously described (Calabrese et al., 2014). Continuous secondary efficacy variables were analyzed using analysis of variance and categorical secondary variables were analyzed by Cochran-Mantel-Haenszel test, as previously described (Calabrese et al., 2014). Safety and tolerability were monitored throughout the study.

\section{Results and discussion \\ Participants}

Of the 656 patients with bipolar I depression who were screened, 399 were enrolled; 200 were randomized to receive adjunctive armodafinil $150 \mathrm{mg} /$ day and 199 were randomized to receive adjunctive placebo (Fig. 1). Baseline demographic and clinical characteristics were statistically similar between treatment groups (Table 1). The efficacy analysis included a total of 393 patients (adjunctive armodafinil $150 \mathrm{mg} /$ day, $n=197$; adjunctive placebo, $n=196$ ); the safety analysis set included 398 patients (adjunctive armodafinil $150 \mathrm{mg} /$ day, $n=200$; adjunctive placebo, $n=198)$. A total of $63(16 \%)$ patients withdrew from the study (31 (16\%) receiving adjunctive armodafinil and $32(16 \%)$ receiving adjunctive placebo). At baseline, most patients $(n=308,77 \%)$ were taking only one mood stabilizer/antipsychotic. Valproate, lamotrigine, and risperidone were the most common mood stabilizer/antipsychotic treatments taken as monotherapy (Table 2). At baseline, depression scores were consistent with moderate to severe depression and were statistically similar between treatment groups.

A total of 17 patients discontinued early due to AEs (adjunctive armodafinil $150 \mathrm{mg} /$ day, 7/200 (4 \%); adjunctive placebo, 10/199 (5\%)). Anxiety and bipolar I disorder were the only AEs that caused discontinuation in $>1$ patient; anxiety led to treatment discontinuation in 3 ( $2 \%)$ of those treated with adjunctive armodafinil and 0 treated with adjunctive placebo; 2 patients taking adjunctive armodafinil and 0 patients taking adjunctive placebo discontinued due to bipolar I disorder.

\section{Efficacy \\ Primary efficacy}

Baseline mean IDS- $\mathrm{C}_{30}$ scores were 42.4 in the adjunctive armodafinil group and 43.3 in the adjunctive placebo group. The least-square (LS) mean and standard error of the LS mean (SEM) change from baseline to week 8 on the IDS- $\mathrm{C}_{30}$ (primary efficacy parameter) for armodafinil versus placebo $(-20.8 \pm 0.99$ vs $-19.4 \pm 0.99)$ in the adjunctive treatment of bipolar I depression was not statistically significant $(P=0.27)$.

\section{Secondary efficacy}

Although not adjusted for multiple comparisons, several secondary efficacy outcomes suggested advantages in favor of adjunctive armodafinil (Table 3). Specifically, statistically significant differences for the IDS- $\mathrm{C}_{30}$ secondary variables in favor of adjunctive armodafinil $150 \mathrm{mg} /$ day over adjunctive placebo included proportion of IDS- $\mathrm{C}_{30}$ responders at week 6 (41 vs $29 \%, P=0.018$ ), week 7 (51 vs $39 \%, P=0.015)$, and week 8 (56 vs $46 \%, P=0.039$ ) (Fig. 2, left); proportion of IDS-C $\mathrm{C}_{30}$ remitters at week 8 (26 vs $15 \%, P=0.011$ ) and endpoint (22 vs $13 \%, P=0.011$ ) (Fig. 2 , right); and mean change from baseline in IDS- $C_{30}$ total score at week $7(P<0.05)$. In addition, statistically significant differences in favor of adjunctive armodafinil were observed for the mean change from baseline in CGI-S rating for depression at week 6 $(P<0.03)$, week $8 \quad(P<0.02)$, and endpoint $(P<0.04)$ and in mean change from baseline in GAF scores, indicating improvement in patient functioning, at week 4 $(P<0.02)$, week $8(P<0.002)$, and endpoint $(P<0.01)$.

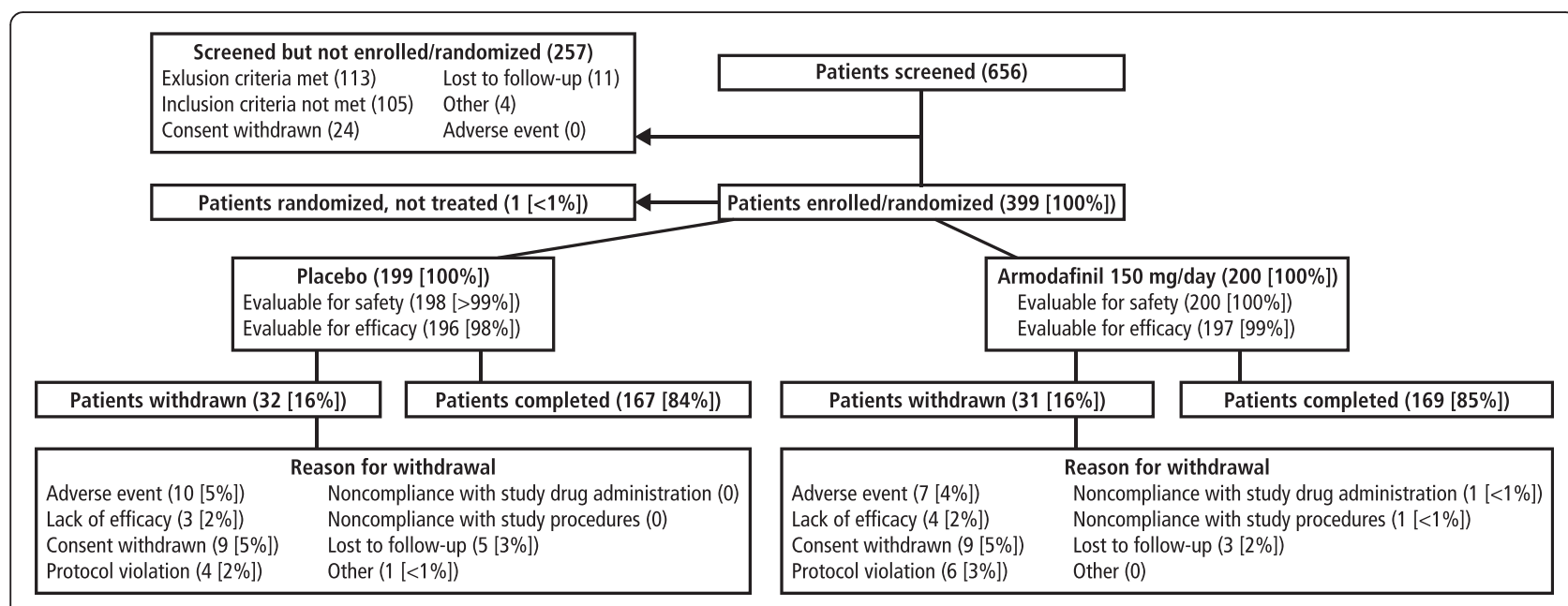

Fig. 1 Patient flow 
Table 1 Baseline demographics and clinical characteristics

\begin{tabular}{|c|c|c|}
\hline Characteristic & $\begin{array}{l}\text { Placebo } \\
n=199\end{array}$ & $\begin{array}{l}\text { Armodafinil } 150 \mathrm{mg} / \text { day } \\
n=200\end{array}$ \\
\hline Age, years, mean (SD) & $43.7(11.6)$ & $45.3(11.3)$ \\
\hline Males, $n(\%)$ & $78(39)$ & $80(40)$ \\
\hline \multicolumn{3}{|l|}{ Race, $n(\%)$} \\
\hline White & $176(88)$ & $182(91)$ \\
\hline Black & $16(8)$ & $14(7)$ \\
\hline Other & $7(4)$ & $4(2)$ \\
\hline Weight, kg, mean (SD) & $81.2(17.5)$ & $80.7(17.5)$ \\
\hline IDS- $C_{30}$ total score, mean (SD) & $43.3(7.7)$ & $42.4(7.7)$ \\
\hline QIDS- $C_{16}$ total score, mean (SD) & $16.8(2.7)$ & $16.1(2.7)$ \\
\hline CGI-S score, mean (SD) & $4.6(0.6)$ & $4.6(0.7)$ \\
\hline GAF score, mean (SD) & $54.8(6.7)$ & $53.6(8.9)$ \\
\hline \multicolumn{3}{|l|}{ C-SSRS } \\
\hline \multicolumn{3}{|l|}{ Suicidal behavior, $n$ (\%) } \\
\hline Suicidal behavior, actual attempt & $20(10)$ & $21(11)$ \\
\hline $\begin{array}{l}\text { Non-suicidal self-injurious } \\
\text { behavior }\end{array}$ & $7(4)$ & $6(3)$ \\
\hline Interrupted attempt & $3(2)$ & $2(1)$ \\
\hline Aborted attempt & $4(2)$ & $4(2)$ \\
\hline Suicidal behavior & $4(2)$ & $4(2)$ \\
\hline Preparatory acts or behavior & $5(3)$ & $6(3)$ \\
\hline \multicolumn{3}{|l|}{ Suicidal ideation, $n(\%)$} \\
\hline Suicidal ideation, wish to be dead & $27(14)$ & $37(19)$ \\
\hline $\begin{array}{l}\text { Non-specific active suicidal } \\
\text { thoughts }\end{array}$ & $16(8)$ & $24(12)$ \\
\hline $\begin{array}{l}\text { Any methods (no plan) without } \\
\text { intent to act }\end{array}$ & $12(6)$ & $20(10)$ \\
\hline $\begin{array}{l}\text { Some intent to act without } \\
\text { specific plan }\end{array}$ & $9(5)$ & $14(7)$ \\
\hline Specific plan and intent & $10(5)$ & $15(8)$ \\
\hline YMRS total score, mean (SD) & $3.7(2.1)$ & $3.7(2.2)$ \\
\hline HAM-A total score, mean (SD) & $12.6(2.8)$ & $12.5(2.9)$ \\
\hline ISI total score, mean (SD) & $16.1(5.2)$ & $15.7(5.5)$ \\
\hline
\end{tabular}

CGI-S Clinical Global Impression of Severity of Illness, C-SSRS-SLV ColumbiaSuicide Severity Rating Scale-Since Last Visit, GAF Global Assessment of Functioning, HAM-A Hamilton Anxiety Scale, IDS- $\mathrm{C}_{30} 30$-Item Inventory of Depressive Symptomatology-Clinician-Rated, ISI Insomnia Severity Index, QIDS-C ${ }_{16}$ 16-Item Quick Inventory of Depressive Symptomatology-ClinicianRated, SD standard deviation, YMRS Young Mania Rating Scale

There were numeric, but not statistically significant, findings favoring adjunctive armodafinil versus adjunctive placebo on the LS mean \pm SEM change from baseline to endpoint in IDS- $C_{30}$ total score $(-18.2 \pm 1.23$ vs $-17.1 \pm$ 1.23), QIDS- $C_{16}$ total score $(-7.1 \pm 0.49$ vs $-7.0 \pm 0.49)$, and IDS- $\mathrm{C}_{30}$ response at endpoint ( 49 vs $\left.41 \%\right)$. There were no statistically significant differences between groups in the proportion of CGI-S responders at any assessment point.
Table 2 Concomitant mood stabilizers

\begin{tabular}{|c|c|c|}
\hline $\begin{array}{l}\text { Mood stabilizer at baseline, } \\
n(\%)\end{array}$ & $\begin{array}{l}\text { Placebo } \\
n=199\end{array}$ & $\begin{array}{l}\text { Armodafinil } 150 \mathrm{mg} / \text { day } \\
n=200\end{array}$ \\
\hline Patients taking one mood stabilizer & $150(75)$ & $158(79)$ \\
\hline Aripiprazole & $16(8)$ & $9(5)$ \\
\hline Lamotrigine & $26(13)$ & $28(14)$ \\
\hline Lithium & $12(6)$ & $18(9)$ \\
\hline Olanzapine & $23(12)$ & $25(13)$ \\
\hline Quetiapine & $18(9)$ & $26(13)$ \\
\hline Risperidone & $29(15)$ & $24(12)$ \\
\hline Valproic acid & $26(13)$ & $28(14)$ \\
\hline Patients taking two mood stabilizers & $45(23)$ & $38(19)$ \\
\hline Aripiprazole + lamotrigine & $4(2)$ & $1(<1)$ \\
\hline Lamotrigine + quetiapine & $7(4)$ & $1(<1)$ \\
\hline Lamotrigine + ziprasidone & $1(<1)$ & $1(<1)$ \\
\hline Lithium + aripiprazole & $1(<1)$ & 0 \\
\hline Lithium + lamotrigine & $3(2)$ & $2(1)$ \\
\hline Lithium + olanzapine & $2(1)$ & 0 \\
\hline Lithium + quetiapine & $4(2)$ & $3(2)$ \\
\hline Lithium + risperidone & $1(<1)$ & $1(<1)$ \\
\hline Lithium + valproic acid & $2(1)$ & $1(<1)$ \\
\hline Lithium + ziprasidone & $3(2)$ & $1(<1)$ \\
\hline Olanzapine + lamotrigine & $1(<1)$ & $1(<1)$ \\
\hline Olanzapine + quetiapine & $1(<1)$ & 0 \\
\hline Valproic acid + aripiprazole & 0 & $2(1)$ \\
\hline Valproic acid + lamotrigine & $2(1)$ & 0 \\
\hline Valproic acid + olanzapine & $5(3)$ & $8(4)$ \\
\hline Valproic acid + quetiapine & $5(3)$ & $9(5)$ \\
\hline Valproic acid + risperidone & $3(2)$ & $5(3)$ \\
\hline Valproic acid + ziprasidone & 0 & $2(1)$ \\
\hline $\begin{array}{l}\text { Patients taking three mood } \\
\text { stabilizers }\end{array}$ & $1(<1)$ & $3(2)$ \\
\hline $\begin{array}{l}\text { Lithium + lamotrigine + } \\
\text { ziprasidone }\end{array}$ & 0 & $1(<1)$ \\
\hline $\begin{array}{l}\text { Valproic acid + aripiprazole + } \\
\text { risperidone }\end{array}$ & 0 & $1(<1)$ \\
\hline $\begin{array}{l}\text { Valproic acid + olanzapine + } \\
\text { lamotrigine }\end{array}$ & 0 & $1(<1)$ \\
\hline $\begin{array}{l}\text { Valproic acid + risperidone }+ \\
\text { quetiapine }\end{array}$ & $1(<1)$ & 0 \\
\hline $\begin{array}{l}\text { Patients with mood stabilizer } \\
\text { unknown }\end{array}$ & $3(2)$ & $1(<1)$ \\
\hline
\end{tabular}

\section{Safety}

Adjunctive armodafinil $150 \mathrm{mg} /$ day in bipolar I depression was generally well tolerated. Overall, 89 (45\%) patients receiving adjunctive armodafinil and 71 (36\%) patients receiving adjunctive placebo experienced $\geq 1 \mathrm{AE}$. Of these, 53 (27 \%) AEs with adjunctive armodafinil and 32 (16\%) with adjunctive placebo were considered treatment- 
Table 3 Secondary efficacy parameters, full analysis set

\begin{tabular}{lccc}
\hline Time point, statistic & $\begin{array}{c}\text { Placebo } \\
n=196\end{array}$ & $\begin{array}{l}\text { Armodafinil } 150 \mathrm{mg} / \text { day } \\
n=197\end{array}$ & $P$ value \\
\hline LSM change from baseline in IDS- $C_{30}$ total score & \\
Week 1 & -6.1 & -5.5 & 0.3025 \\
Week 2 & -10.4 & -9.3 & 0.1940 \\
Week 4 & -12.3 & -12.5 & 0.8481 \\
Week 6 & -14.2 & -16.1 & 0.0926 \\
Week 7* & -16.0 & -18.3 & 0.0492 \\
Week 8 & -17.7 & -19.6 & 0.1174 \\
Endpoint & -18.3 & -19.5 & 0.3526
\end{tabular}

LSM change from baseline in QIDS- $C_{16}$ total score

$\begin{array}{llll}\text { Week 1 } & -2.6 & -2.4 & 0.3858 \\ \text { Week 2* } & -4.5 & -3.8 & 0.0387 \\ \text { Week 4 } & -5.2 & -5.2 & 0.9978 \\ \text { Week 6 } & -6.0 & -6.5 & 0.3024 \\ \text { Week 7 } & -6.7 & -7.4 & 0.1530 \\ \text { Week 8 } & -7.4 & -7.7 & 0.5471 \\ \text { Endpoint } & -7.0 & -7.1 & 0.7626\end{array}$

LSM change from baseline in CGI-S score

$\begin{array}{llll}\text { Week 1 } & -0.2 & -0.2 & 0.4497 \\ \text { Week 2 } & -0.5 & -0.5 & 0.9625 \\ \text { Week 4 } & -0.7 & -0.8 & 0.1467 \\ \text { Week 6* } & -0.9 & -1.2 & 0.0226 \\ \text { Week 7 } & -1.1 & -1.3 & 0.0757 \\ \text { Week 8* } & -1.2 & -1.5 & 0.0159 \\ \text { Endpoint } & -1.1 & -1.3 & 0.0320 \\ \text { Proportion of CGI-S responders, n (\%) } & \\ \text { Week 1 } & 4(2) & 4(2) & \\ \text { Week 2 } & 15(8) & 16(8) & 0.9939 \\ \text { Week 4 } & 28(15) & 36(20) & 0.8927 \\ \text { Week 6 } & 44(26) & 56(33) & 0.2585 \\ \text { Week 7 } & 55(32) & 68(40) & 0.1350 \\ \text { Week 8 } & 66(40) & 84(50) & 0.1031 \\ \text { Endpoint } & 67(34) & 86(44) & 0.0516 \\ \text { Week 4* } & 5.3 & 7.7 & 0.0503 \\ \text { Week 8** } & 11.4 & 15.2 & 0.0113 \\ \text { Endpoint } & 10.4 & 13.5 & 0.0012 \\ \text { CGI-SClnic } & 15.4 & 0.0066\end{array}$

CGI-S Clinical Global Impression of Severity of Illness, GAF Global Assessment of Functioning, IDS- $C_{30} 30$-Item Inventory of Depressive Symptomatology-Clinician-Rated, $L S M$ least-square mean, QIDS- $C_{16}$ 16-Item Quick Inventory of Depressive Symptomatology-Clinician-Rated ${ }^{*} P<0.05$. ${ }^{* *} P<0.01$.

${ }^{a}$ The denominator for calculating the percentages at each visit is the number of patients with a non-missing value at that visit. A responder is a patient with a decrease of at least 2 points in severity from baseline in CGI-S rating for depression. The $P$ value for the treatment comparison is from a Cochran-Mantel-Haenszel test, stratified by concurrent mood-stabilizing medication and region of the world related. Only 2 AEs were observed at a rate exceeding $5 \%$ in either treatment group: headache in 29 (15\%) with adjunctive armodafinil vs $15(8 \%)$ with adjunctive placebo and nausea in $12(6 \%)$ with adjunctive armodafinil vs 7 (4\%) with adjunctive placebo. The majority of AEs were mild (in $53(27 \%)$ vs $47(24 \%)$ ) or moderate (in $32(16 \%)$ vs $20(10 \%))$ in severity for adjunctive armodafinil vs adjunctive placebo, respectively.

Rates of AEs causing withdrawals and incidences of SAEs were similar between groups. Withdrawals due to AEs occurred in $7(4 \%)$ patients taking adjunctive armodafinil and $10(5 \%)$ patients taking adjunctive placebo. Serious AEs occurred in $5(3 \%)$ patients taking adjunctive armodafinil and 6 (3\%) patients taking adjunctive placebo; no single SAE occurred in $>1$ patient. No deaths occurred in either treatment group. At study endpoint, there were no clinically significant differences in mean changes from baseline in serum chemistries, lipid profiles, and hematologic or urinalysis parameters noted between the two treatment groups. There were no clinically significant changes in vital signs or electrocardiograms in either treatment group.

There were few changes from baseline to endpoint in suicidal ideation and behavior as assessed by C-SSRS-SLV. In the adjunctive armodafinil group, 1 patient had an SAE coded using the Medical Dictionary for Regulatory Activities preferred term of "bipolar I disorder" (specifically, exacerbation of bipolar disorder (mixed episode with psychotic symptoms)), accompanied by a suicide attempt, with no substantive threat to life; both SAEs resolved with no residual effect. One patient taking adjunctive placebo had an SAE of suicidal ideation, which resolved without any sequelae.

Mean changes in YMRS, HAM-A, and ISI total scores from baseline to endpoint were statistically similar with adjunctive armodafinil vs adjunctive placebo, respectively, as follows: YMRS ( -0.9 vs -1.0$)$, HAM-A $(-4.3$ vs -4.2$)$, and ISI $(-7.1$ vs -7.0$)$.

The following AE rate differences were observed between adjunctive armodafinil and adjunctive placebo, respectively: emergence of hypomania $(0(0 \%)$ vs $1(<1 \%))$, anxiety $(8(4 \%)$ vs $5(3 \%))$, insomnia $(6(3 \%)$ vs $4(2 \%))$, sedation/somnolence (2 (1\%) vs $2(1 \%))$, and $\geq 7 \%$ weight gain $(4(2 \%)$ vs $9(5 \%))$.

\section{Discussion}

There is a compelling need for effective, well-tolerated treatments for bipolar I depression, for use adjunctively or as monotherapy. Despite the clinical practice of adding adjunctive treatments to ongoing maintenance medications when an episode of bipolar I depression emerges, there is little adequately controlled evidence to inform such clinical decisions. In fact, only one agent, lurasidone, has been approved by the FDA for 


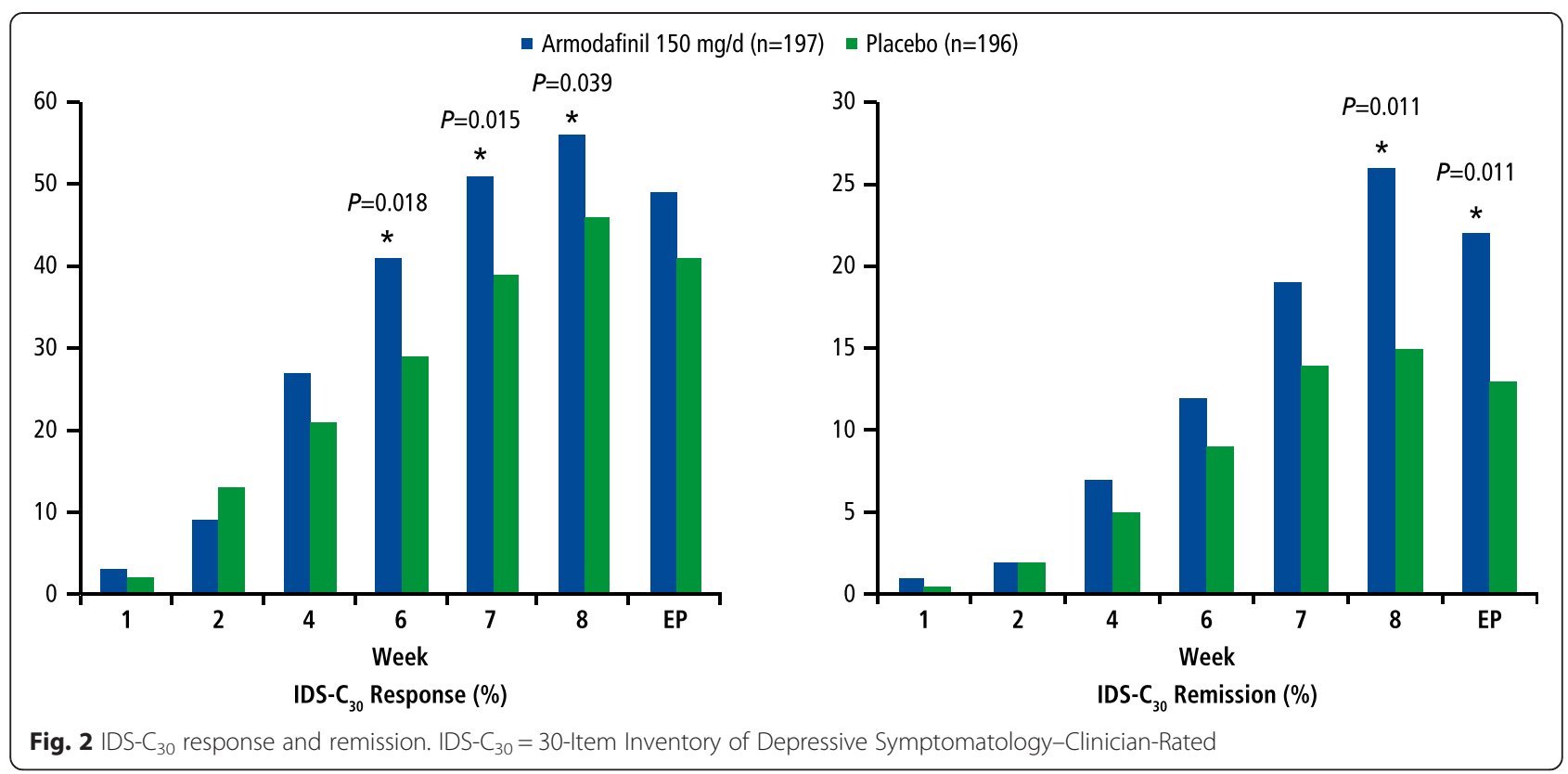

adjunctive use (combined with lithium or valproate) for bipolar I depression (Latuda [package insert], 2013).

A phase 2 study (Calabrese et al., 2010) and two subsequent phase 3 studies (Calabrese et al., 2014; Ketter et al., 2015) previously evaluated the efficacy and safety of adjunctive armodafinil treatment for depressive symptoms in bipolar I disorder, both using the same primary efficacy measure (IDS- $\mathrm{C}_{30}$ ). Conflicting findings from the first two phase 3 studies warranted additional research. In the current study, the adjunctive use of armodafinil $150 \mathrm{mg} /$ day for bipolar I depression provided a numerical but nonsignificant difference vs placebo on the primary outcome (reduction of depressive symptomatology as measured by the IDS- $\mathrm{C}_{30}$ total score). The negative primary outcome result of this study was similar to that of the similarly designed second phase 3 study (Ketter et al., 2015) and did not support or confirm the statistically significant primary outcome finding from the initial phase 3 study (Calabrese et al., 2014). However, in the current study (like the first study and unlike the second study), adjunctive armodafinil $150 \mathrm{mg} /$ day compared with placebo yielded statistically significant benefits for several secondary outcomes, including the proportion of IDS- $C_{30}$ responders at weeks 6,7 , and 8 , proportion of IDS- $\mathrm{C}_{30}$ remitters at week 8 and endpoint, and mean change from baseline in IDS- $\mathrm{C}_{30}$ total score at week 7 . In addition, mean change from baseline in the CGI-S rating for depression (indicating improvement in depressive symptomatology) was statistically significant at weeks 6 and 8, and at endpoint, and mean change from baseline in the GAF score (indicating improvement in patient functioning) was statistically significant at weeks 4 and 8 , and at endpoint. In all 3 studies, adjunctive armodafinil was consistently well tolerated, with no clinically relevant differences vs adjunctive placebo for the emergence of hypomania, insomnia, anxiety, sedation/somnolence, or $\geq 7 \%$ weight gain.

Some patients with bipolar disorder may be candidates for combination therapy to manage manic, hypomanic, mixed, depressed, and/or cycling related mood symptoms. In contrast to most regulatory nonmonotherapy bipolar disorder clinical trials, which limit adjunctive treatments to lithium, valproate, and only very recently lamotrigine or one atypical antipsychotic, this study allowed 29 different combinations of moodstabilizing treatments prior to study randomization (see Table 2). The assay sensitivity (i.e., drug-placebo separation) of this study was potentially limited by what was identified a priori as a study design strength (i.e., potential generalizability and community translation). A second challenge overall is the design of a study for a novel compound that is not considered a "mood stabilizer" where the trial design template has conventionally been designed for atypical antipsychotic mood stabilizers (i.e., lurasidone, olanzapine/fluoxetine, and quetiapine).

\section{Conclusions}

In this 8-week study marked by significant therapeutic heterogeneity, adjunctive armodafinil $150 \mathrm{mg} /$ day yielded a numerically greater, but not statistically significant, improvement on the primary efficacy outcome measure compared with placebo in treatment of bipolar I depression. Several secondary outcomes favoring armodafinil at endpoint, including a significantly higher remission rate, and paralleled by significant global and 
functional improvements rated by clinical investigators, indicate that adjunctive armodafinil provided a reduction of depressive symptoms in a subset of patients with bipolar I depression vs placebo. Adjunctive armodafinil was generally well tolerated in this study, with rates of anxiety, insomnia, sedation/somnolence, and weight gain similar to those observed with adjunctive placebo.

This investigation adds to the evidence from two prior phase 3 studies of similar design, in which armodafinil $150 \mathrm{mg} /$ day, adjunctive to protocol-allowed mood stabilizers, provided statistically significant improvement in at least some measures of depressive symptoms associated with bipolar I disorder (Study 3071; NCT01072929 and Study 3072; NCT01072630) (Calabrese et al. 2014; Ketter et al. 2015). Although armodafinil was generally well tolerated in this and prior phase 3 studies, the lack of statistically significant efficacy on the primary outcome in two of three studies has led to the discontinuation of the development program for adjunctive armodafinil in bipolar I depression. Additional research is warranted and necessary to better identify clinical predictors (e.g., atypical depressive symptoms, specific combinations of therapeutic agents) that would provide optimized and individualized therapeutics for bipolar depression.

\section{Abbreviations \\ AE: adverse event; CGI-S: Clinical Global Impression of Severity of IIIness; C-SSRS-SLV: Columbia-Suicide Severity Rating Scale-Since Last Visit; GAF: Global Assessment of Functioning; HAM-A: Hamilton Anxiety Scale; IDS- $C_{30}$ : 30-Item Inventory of Depressive Symptomatology-Clinician- Rated; ISI: Insomnia Severity Index; LSM: least-square mean; MMRM: mixed-model repeated measures; QIDS- $C_{16}$ : 16-Item Quick Inventory of Depressive Symptomatology-Clinician-Rated; SAE: serious adverse event; SD: standard deviation; SCID-CT: Structured Clinical Interview for DSM-IV, Clinical Trials; YMRS: Young Mania Rating Scale.}

\section{Competing interests}

MAF has received grant support from Assurex, Janssen Research \& Development, Mayo Foundation, Myriad, the National Institute of Alcohol Abuse and Alcoholism (NIAAA), the National Institute of Mental Health (NIMH), and Pfizer, served as a Consultant for (Mayo)-Janssen Research \& Development, LLC, Mitsubishi Tanabe Pharma Corporation, Myriad, Sunovion, Supernus Pharmaceuticals, and Teva Pharmaceuticals. Mayo Clinic has a financial interest in Assurex Health; this technology is not referenced in this publication/presentation.

JA has served as a paid consultant for AstraZeneca, BTG International, Forest Laboratories, Mallinckrodt Pharmaceuticals, MannKind Corporation, Orbis Clinical, ReSearch Pharmaceutical Services, Sermo, Shire, Sucampo Pharmaceuticals, Taisho Pharmaceuticals, Teva Pharmaceuticals, ViroPharma, and Yoh.

MB has received grants from the American Foundation for Suicide Prevention, Bundesministeriums für Bildung und Forschung (BMBF), Deutsche Forschungsgemeinschaft (DFG), the European Commission, the National Alliance for Research on Schizophrenia and Depression (NARSAD), and Stanley Medical Research Institute and received honoraria as a board member, speakers bureau member, or consultant for AstraZeneca, Ferrer International, Janssen, Lilly, Lundbeck, Otsuka, Pfizer, GSK, Servier, and Takeda. CA has received research funding from Merck and Forest and served as a speaker and received honoraria from Sunovion. He has received grant support from the National Institute of Mental Health and non-profit foundations.
RY is employed by Teva Pharmaceuticals (formerly Cephalon) and has stock ownership/options with Teva Pharmaceuticals (formerly Cephalon). TAK has received research funding from the Agency for Healthcare Research and Quality, AstraZeneca, Eli Lilly, Pfizer, Sepracor, Sunovion, and Teva Pharmaceuticals (formerly Cephalon), served as an advisor/consultant to Allergan, Avanir, Forest, Janssen, Merck, Sepracor, Sunovion, Quintiles, and Teva Pharmaceuticals (formerly Cephalon), and served as a speaker and received honoraria from Abbott, AstraZeneca, GlaxoSmithKline, Janssen, Johnson \& Johnson, and Otsuka. TAK's spouse, Nzeera Ketter, MD, is employed by Janssen, Johnson \& Johnson and has stock ownership/options with Janssen, Johnson \& Johnson.

\section{Authors' contributions}

TAK and CA participated in the design of the study and revised the manuscript. MB helped draft the manuscript at various stages of development. RY participated in the design of the study and performed the statistical analysis. MAF contributed/consulted to initial study design, manuscript draft, and revisions and gave final approval for submission. JA assisted in monitoring of the study, participated in data review, and developed and reviewed the manuscript. All authors read and approved the final manuscript.

\section{Acknowledgements}

Medical writing assistance was provided by John H. Simmons, MD, of Peloton Advantage and was funded by the Teva Branded Pharmaceutical Products R \& D, Inc. (Frazer, PA). Teva provided a full review of the article.

\section{Funding}

This study was sponsored by the Teva Pharmaceutical Industries, Ltd. (Petah Tikva, Israel).

\section{Author details}

'Department of Psychiatry, Mayo Clinic, 200 First Street SW, Rochester, MN 55901, USA. ${ }^{2}$ Teva Pharmaceuticals, Frazer, PA, USA. ${ }^{3}$ University Hospital Carl Gustav Carus, Technische Universität Dresden, Dresden, Germany. ${ }^{4}$ University of Cincinnati, Cincinnati, OH, USA. ${ }^{5}$ Stanford University, Stanford, CA, USA.

Received: 6 May 2015 Accepted: 23 July 2015

Published online: 02 September 2015

\section{References}

American Psychiatric Association. Diagnostic and statistical manual of mental disorders, text revision: DSM-IV-TR. 4th ed. Washington, DC: American Psychiatric Association; 2000.

Bastien $\mathrm{CH}$, Vallieres A, Morin CM. Validation of the Insomnia Severity Index as an outcome measure for insomnia research. Sleep Med. 2001;2:297-307.

Bauer MS, Kirk GF, Gavin C, Williford WO. Determinants of functional outcome and healthcare costs in bipolar disorder: a high-intensity follow-up study. J Affect Disord. 2001;65:231-41.

Calabrese JR, Hirschfeld RM, Frye MA, Reed ML. Impact of depressive symptoms compared with manic symptoms in bipolar disorder: results of a U.S. community-based sample. J Clin Psychiatry. 2004;65:1499-504.

Calabrese JR, Ketter TA, Youakim JM, Tiller JM, Yang R, Frye MA. Adjunctive armodafinil for major depressive episodes associated with bipolar I disorder: a randomized, multicenter, double-blind, placebo-controlled, proof-of-concept study. J Clin Psychiatry. 2010;71:1363-70.

Calabrese JR, Frye MA, Yang R, Ketter TA, for the Armodafinil Treatment Trial Study Network. Efficacy and safety of armodafinil for the adjunctive treatment of major depressive episodes associated with bipolar I disorder: a randomized, double-blind, placebo-controlled; multicenter trial. J Clin Psychiatry. 2014;75:1054-61.

First MB, Williams JBW, Spitzer RL, Gibbon M. Structured clinical interview for DSM-IV-TR axis I disorders, clinical trials version (SCID-CT). New York: Biometrics Research, New York State Psychiatric Institute; 2007.

Frye MA, Grunze H, Suppes T, McElroy SL, Keck PE, Walden J, et al. A placebocontrolled evaluation of adjunctive modafinil in the treatment of bipolar depression. Am J Psychiatry. 2007;164:1242-9.

Hamilton Anxiety Rating Scale (HAM-A). 2011; UF MedConsults. Available at: http://dcf.psychiatry.ufl.edu/files/2011/05/HAMILTON-ANXIETY.pdf. Accessed: July 20, 2015. 
Judd LL, Akiskal HS, Schettler PJ, Endicott J, Maser J, Solomon DA, et al. The long-term natural history of the weekly symptomatic status of bipolar I disorder. Arch Gen Psychiatry. 2002:59:530-7.

Kessler RC, Chiu WT, Demler O, Merikangas KR, Walters EE. Prevalence, severity, and comorbidity of 12-month DSM-IV disorders in the National Comorbidity Survey Replication. Arch Gen Psychiatry. 2005;62:617-27.

Ketter TA, Yang R, Frye MA. Adjunctive armodafinil for major depressive episodes associated with bipolar I disorder. J Affect Disord. 2015;181:87-91.

Kupka RW, Altshuler LL, Nolen WA, Suppes T, Luckenbaugh DA, Leverich GS, et al. Three times more days depressed than manic or hypomanic in both bipolar I and bipolar || disorder. Bipolar Disord. 2007;9:531-5.

Latuda [package insert]. Marlborough, MA: Sunovion Pharmaceuticals Inc., 2013.

Lombardo I, Sachs G, Kolluri S, Kremer C, Yang R. Two 6-week, randomized, double-blind, placebo-controlled studies of ziprasidone in outpatients with bipolar I depression: did baseline characteristics impact trial outcome? J Clin Psychopharmacol. 2012;32:470-8.

Merikangas KR, Akiskal HS, Angst J, Greenberg PE, Hirschfeld RMA, Petukhova $M$, et al. Lifetime and 12-month prevalence of bipolar spectrum disorder in the National Comorbidity Survey replication. Arch Gen Psychiatry. 2007; 64:543-52.

Merikangas KR, Jin R, He JP, Kessler RC, Lee S, Sampson NA, et al. Prevalence and correlates of bipolar spectrum disorder in the world mental health survey initiative. Arch Gen Psychiatry. 2011;68:241-51.

Nuvigil [package insert]. North Wales, PA: Teva Pharmaceuticals USA, Inc., 2013.

Posner K, Brent D, Lucas C, Gould M, Stanley B, Brown G, Fisher P, Zelazny J, Burke A, Oquendo M, Mann J. Columbia-Suicide Severity Rating Scale (C-SSRS) Since Last Visit. 2009; Columbia University Medical Center. Available at: http://www.cssrs.columbia.edu/docs/C-SSRS_1-14-09-Since_Last_Visit_Clinical.pdf. Accessed: July 20, 2015.

Rush AJ, Carmody T, Reimitz PE. The inventory of depressive symptomatology (IDS): clinician (C-IDS) and self-report (IDS-SR) ratings of depressive symptoms. Int J Meth Psychiatr Res. 2000;9:45-59.

Rush AJ, Trivedi MH, Ibrahim HM, Carmody TJ, Arnow B, Klein DN, et al. The 16-Item Quick Inventory of Depressive Symptomatology (QIDS), clinician rating (QIDS-C), and self-report (QIDS-SR): a psychometric evaluation in patients with chronic major depression. Biol Psychiatry. 2003;54:573-83.

Sachs GS, Ice KS, Chappell PB, Schwartz JH, Gurtovaya O, Vandenburg DG, et al. Efficacy and safety of adjunctive oral ziprasidone for acute treatment of depression in patients with bipolar I disorder: a randomized, double-blind, placebo-controlled trial. J Clin Psychiatry. 2011;72:1413-22.

Thase ME, Jonas A, Khan A, Bowden CL, Wu X, McQuade RD, et al. Aripiprazole monotherapy in nonpsychotic bipolar I depression: results of 2 randomized, placebo-controlled studies. J Clin Psychopharmacol. 2008;28:13-20.

Young RC, Biggs JT, Ziegler VE, Meyer DA. A rating scale for mania: reliability, validity and sensitivity. Br J Psychiatry. 1978;133:429-35.

\section{Submit your manuscript to a SpringerOpen ${ }^{\odot}$ journal and benefit from:}

- Convenient online submission

Rigorous peer review

- Immediate publication on acceptance

- Open access: articles freely available online

- High visibility within the field

- Retaining the copyright to your article

Submit your next manuscript at springeropen.com 